\title{
Safflower yellow pigment and Sanqi Panax notoginseng in the treatment of acute cerebral infarction: a systematic review, meta-analysis, and cost-effectiveness analysis
}

\author{
Yang $\mathrm{Li}^{1 \#}$, Yan Lin ${ }^{2 \#}$, Zhixiang Shi ${ }^{3}$, Yuxuan $\mathrm{Hu}^{1}$, Taihang Shao ${ }^{1}$, Mengyuan $\mathrm{Li}^{2}$, Jiawen Zhu ${ }^{4}$, Lizhou Sun ${ }^{5}$, \\ Feng Chang ${ }^{1}$
}

${ }^{1}$ School of International Pharmaceutical Business, China Pharmaceutical University, Nanjing, China; ${ }^{2}$ School of Basic Medicine and Clinical Pharmacy, China Pharmaceutical University, Nanjing, China; ${ }^{3}$ School of Foreign Languages, China Pharmaceutical University, Nanjing, China; ${ }^{4}$ Institute of Pharmaceutical Sciences, China Pharmaceutical University, Nanjing, China; ${ }^{5}$ Department of Obstetrics and Gynecology, The First Affiliated Hospital of Nanjing Medical University, Nanjing, China

Contributions: (I) Conception and design: F Chang, Y Li; (II) Administrative support: Y Li, Y Lin, L Sun; (III) Provision of study materials or patients: F Chang, Y Li; (IV) Collection and assembly of data: Y Hu, T Shao, M Li; (V) Data analysis and interpretation: Y Hu, J Zhu; (VI) Manuscript writing: All authors; (VII) Final approval of manuscript: All authors.

\#These authors contributed equally to this work.

Correspondence to: Feng Chang. School of International Pharmaceutical Business, China Pharmaceutical University, Nanjing, China. Email: cfcpu72@163.com; Lizhou Sun. Department of Obstetrics and Gynecology, The First Affiliated Hospital of Nanjing Medical University, Nanjing, China. Email: Lizhou_sun@163.com.

Background: Sanqi Panax notoginseng injection and safflower yellow injection were Chinese traditional medicine injections for the treatment of cardiovascular diseases and were used to treat acute cerebral infarction patients in public hospital widely. The aim of this study was to compare and analyze the published reports of efficacy and safety of Sanqi Panax notoginseng injection and safflower yellow injection for the treatment of acute cerebral infarction. The cost-effectiveness of these drug formulations was also evaluated.

Methods: China National Knowledge Infrastructure (CNKI), Wanfang, SinoMed, VIP, PubMed, Embase, and the Chinese Biomedical Literature (CBM) were searched with the restrictions keywords in Chinese and English between 2006 and 2019 to obtain RCTs. A meta-analysis and a meta-regression analysis were undertaken in Reviewer Manager 5.3 software to compare the efficacy and safety of Sanqi Panax notoginseng and safflower yellow injection. This study used a decision tree model to analyze the cost-effectiveness of the two treatments. The TreeAge Pro software was used to comprehensively evaluate the economics of these medications.

Results: Twelve papers were all randomized controlled trials (RCTs) in which Sanqi Panax notoginseng injection was applied in the control group, while safflower yellow injection was applied in the experimental group and the quality of them were good. The results of the 12 papers were compared, and the total effective rate of the treatment group $(91.18 \%)$ was significant and showed no significant difference with the control group (74.83\%) (RR =1.24, 95\% CI: 1.19, 1.30, $\mathrm{P}<0.00001)$. From the perspective of pharmacoeconomics, compared with Sanqi Panax notoginseng group, the ICER of safflower yellow injection is 3,885.75 RMB. The sensitivity analysis results were consistent with the basic analysis results, indicating that the basic analysis results were relatively stable.

Conclusions: Comparing with Sanqi Panax notoginseng injection, safflower yellow injection and related combination therapy can improve the total effective rate and are safer with fewer adverse reactions. It is also more cost-effective than the use of Sanqi Panax notoginseng injection.

Keywords: Safflower yellow pigment; safflower injection; cerebral infarction; meta-analysis; cost-effectiveness analysis 
Submitted Feb 18, 2021. Accepted for publication Jul 11, 2021.

doi: 10.21037/atm-21-782

View this article at: https://dx.doi.org/10.21037/atm-21-782

\section{Introduction}

Cerebral infarction, also known as ischemic stroke, is a common disease in clinical practice. It is caused by local lesions in the cerebral vessel wall, leading to vasospasm, stenosis, or occlusion, thus causing impaired blood supply to the brain, irreversible damage to the local brain tissue, and ultimately ischemic and hypoxic brain tissue necrosis (1-3). According to the statistics of the World Health Organization (WHO), in 2010 and 2016, cerebral infarction was the second leading cause of death globally, next only to ischemic heart disease. In China, cerebral infarction has been the first leading cause of death, with more than 1.5 million new cases each year (4). The Institute of Health and Economics of the Chinese Ministry of Health has reported that the social and economic burden caused by cerebrovascular disease in China is as much as 20 billion yuan per year, and the incidence of cerebral infarction is the most prevalent subtype, so the disease burden it brings is extreme (5). Because most cerebral infarction have a sudden onset and result in a high disability rate, most patients lack sufficient psychological preparation and develop negative emotions such as fear and anxiety, contributing to a psychological burden (6). In sum, cerebral infarction put extra pressure and burden on patients, their families, and the whole society.

Previous studies have shown that Sanqi Panax notoginseng injection, with notoginoside as the main component, has positive therapeutic effects on cerebral infarction (7). It can also significantly improve the neurological function and quality of life of patients. Safflower yellow injection is mainly composed of the yellow pigment extracted from safflower (Carthamus tinctorius). Previous studies have shown that safflower yellow injection significantly dilates blood vessels, improves microcirculation and anticoagulation and reduces blood viscosity, with a beneficial effect for the treatment of coronary heart disease, myocardial ischemia, hyperviscosity, and ischemic cerebrovascular disease (8-14). At present, Sanqi Panax notoginseng and safflower yellow pigment have been all clinically applied to the treatment of cerebral infarction in public hospitals because they were included in China Medical Insurance Directory. However, there is little information reported on the pharmacoeconomic evaluation and the comparison of efficacy and safety between them. The aim of this study was to review the current evidence on the therapeutic effects of safflower yellow injection and Sanqi Panax notoginseng on cerebral infarction. A cost-effectiveness analysis was constructed based on the decision tree model to evaluate the economics of the two drug regimens. The best clinical treatment plan in terms of effectiveness, safety, and economy must be determined to provide guidance for clinical rational drug use, rational resource allocation and medical cost savings. We present the following article in accordance with the PRISMA reporting checklist (available at https://dx.doi.org/10.21037/atm-21-782).

\section{Methods}

The PRISMA guidelines (15) were used for designing and reporting this study, and this study was in accordance with the ethical guidelines of the Declaration of Helsinki (as revised in 2013) (16). Our PROSPERO registration number is CRD42020167326.

\section{Search strategy}

We searched Chinese database such as China National Knowledge Infrastructure (CNKI), Wanfang, SinoMed, and the Chinese Biomedical Literature (CBM); English database such as PubMed, Embase and web of science from January 2006 to January 2019. The keywords included in the search were "safflower yellow", "acute cerebral infarction", and "Sanqi Panax notoginseng". The references cited by the included studies were also evaluated to locate more relevant studies. The ClinicalTrial.gov database was also polled to include human trial results that had not been reported elsewhere. Grey literatures and ongoing trials were searched. We only searched studies in Chinese and English.

\section{Study selection}

The participants of these studies were patients with acute cerebral infarction. Safflower yellow pigment including safflower yellow injection and safflower injection were the intervention of experiment group. Sanqi Panax 
notoginseng injection was the comparison intervention. The route of administration was intravenous drip. The outcome indicator was the total effective rate and National Institutes of Health Stroke Scale (NIHSS) score. The total effective rate is defined based on the NIHSS score: (I) significantly effective: the clinical symptoms of the patient completely disappeared. Loss of function score is greater than $90 \%$, and no disability occurs: (II) effective: the patient's clinical symptoms have been significantly improved, and the vital signs also have more significant changes, the loss of function score is less than $50 \%$ : the degree of disability is less than 3; (III) invalid: the patient's clinical symptoms and vital signs have not changed, and the condition even worsened.

Study types were limited to RCTs and retrospective trials. Animal experiments and case studies were excluded. Studies with incomplete data and duplicate data were excluded.

\section{Data extraction and quality assessment}

Two authors independently assessed the quality of studies for bias analysis using the Cochrane Handbook for Systematic Reviewers version 5.1.0 risk of bias assessment tool for RCTs (17). The following items were assessed: (I) whether the random method is correct; (II) whether allocation concealment is achieved; (III) whether blinding is used (implementation bias); (IV) blinding of outcome evaluators (measurement bias); (V) completeness of outcome data (follow-up bias); (VI) selective reporting (reporting bias); and (VII) other sources of bias. Each aspect of the content had three levels, including "high", "unclear", and "low". In the cases of disagreement, a third party adjudicated.

\section{Statistical analysis}

Statistical analysis was undertaken in Review Manager 5.3 (Cochrane Collaboration, Copenhagen, Denmark). Odds ratio (OR) was used as the effect indicator for enumeration data, and mean $(\bar{x} \pm s)$ was used as the effect indicator for measurement data. The point estimate and $95 \%$ confidence interval (CI) were given for each effect size. Heterogeneity among the results of the included studies was tested using the $\chi^{2}$ test, with $\mathrm{P}<0.05$ as the test level, and the magnitude of heterogeneity was quantitatively determined in combination with $\mathrm{I}^{2}$. If there was no significant heterogeneity among studies $(\mathrm{P}>0.05$,
$\mathrm{I}^{2}<50 \%$ ), the fixed effects model was selected for effect size combination; if there was heterogeneity among studies $\left(\mathrm{P}<0.05, \mathrm{I}^{2}>50 \%\right)$, the random effects model was used for effect size combination. Publication bias was assessed using funnel plots. At the same time, the Egger and Begger values were calculated using STATA14.0 to judge whether there was bias. $\mathrm{P}>0.05$ was taken as the test level. If $\mathrm{P}>0.05$, it was considered that the meta-analysis was unbiased, otherwise there was bias.

In the evaluation of pharmacoeconomics, costeffectiveness analysis was used to calculate the incremental cost-effectiveness ratio; single-factor sensitivity analysis was used to draw the cyclone chart. Monte Carlo simulation was used for probability sensitivity analysis, and then a cost-effectiveness curve was drawn (18). In this paper, TreeAge2011 software was used to build a decision tree model for cost-effectiveness analysis and sensitivity analysis.

\section{Pharmacoeconomic evaluation}

\section{Research perspective}

This study used cost-effectiveness analysis for pharmacoeconomic comparison, considering the cost from the perspective of patients and calculating the treatment efficiency for patients with cerebral infarction. The years of improvement in quality of life, indirect costs of treatment, and hidden costs that cause inconvenience to patients were not considered, because there is no standard available for reference. Therefore, the direct cost of the drug itself, that is, the price per use, was considered to be the cost. In addition, the effective components of safflower yellow injection and safflower injection in this study are the same, although they are produced by different manufacturers. These data were kept separate in the above meta-analysis, and were been evaluated differently in the cost calculation. The decision indicator of the cost-effectiveness analysis was the incremental cost-effectiveness ratio, and the smaller ratio is indicative that the pharmacoeconomic advantage is better.

\section{Decision tree model}

Model structure: this study used a decision tree model to analyze the cost-effectiveness of Safflower yellow injection and Sanqi Panax notoginseng injection included in the meta-analysis for the treatment of cerebral infarction diseases. The meta-analysis method was used to obtain the effectiveness and safety indicators, and comprehensively 


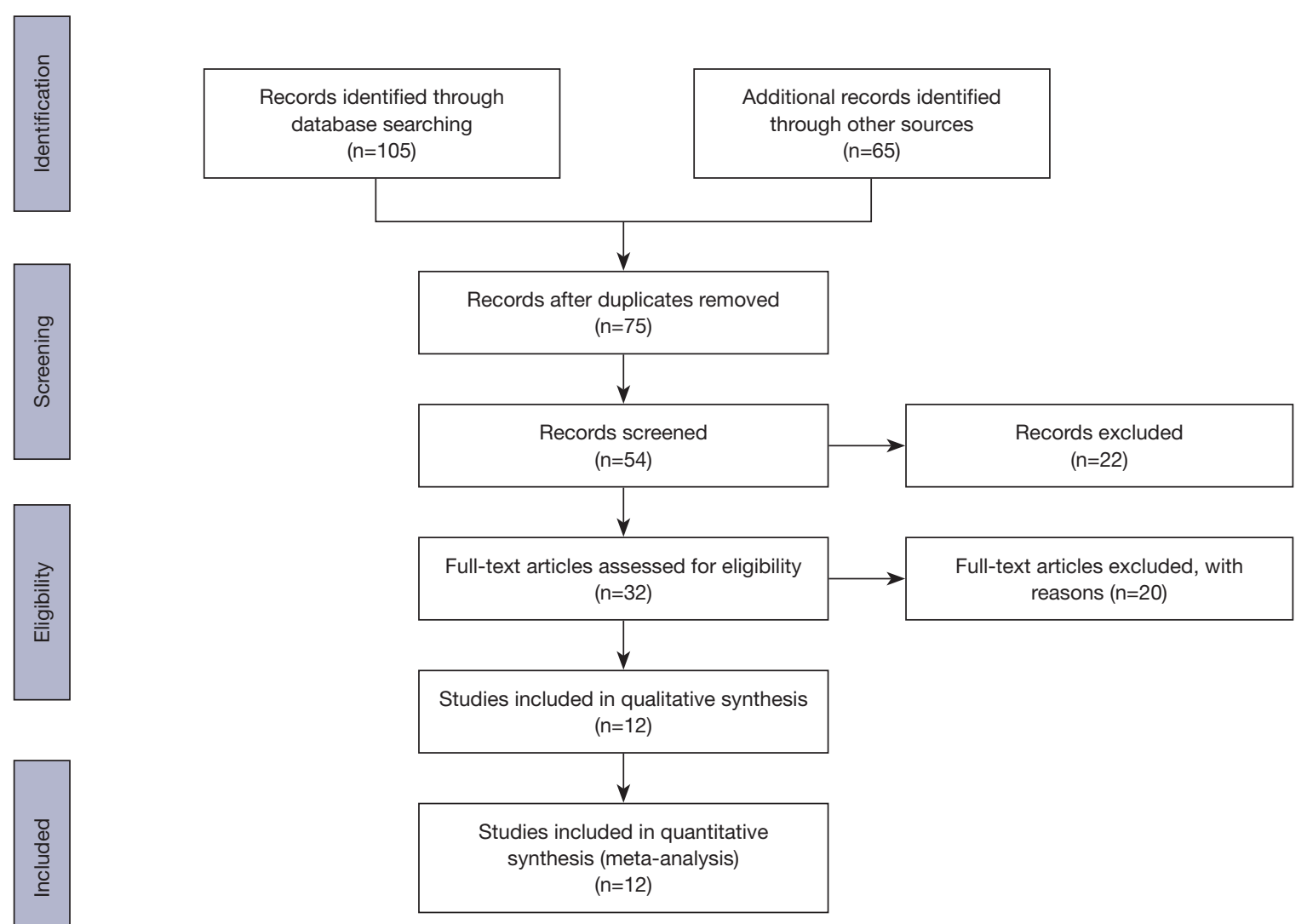

Figure 1 Flow diagram of the literature review.

evaluate the economics of safflower yellow and Sanqi Panax notoginseng. The model mainly evaluated the short-term economics of safflower yellow and Sanqi Panax notoginseng injection. The time span of the model simulation was a course of treatment (14 days).

Effectiveness: in this study, the effective rate of treatment for patients with cerebral infarction was the efficiency index. According to the weight of each study obtained from meta-analysis, the treatment efficiency of cerebral infarction patients in each study was weighted, and the effectiveness included in the pharmacoeconomic evaluation was obtained.

Effectiveness index: judging whether it is effective according to the total effective rate, effective was counted as 1 , invalid or ineffective was counted as 0 .

Cost: this study analyzed cost from the perspective of patients. Since the goal of the patient is to obtain the best prevention and diagnosis results with the least personal expenditure and minimal costs, only the cost to the patient and their family was calculated (19).

\section{Results}

\section{Literature search results}

\section{Literature search and screening results}

Initially, 170 literature reports were identified. After excluding duplicate literatures, 75 literatures were identified. Unrelated papers such as, animal experiments, and case reports were excluded. A refined set of 32 clinical studies on safflower injection for cerebral infarction were remained. After further reading of the full text of each study, all literatures with incomplete data and duplicate data as well as unrelated outcome indicators were excluded. Finally, 12 Chinese literatures reports were included in the study. The literature screening process is shown in Figure 1.

\section{Literature search results and quality evaluation}

The 12 literature reports of RCTs that were included had a total of 1,425 patients, among whom 714 were in the 
experimental group and 711 were in the control group. The literature was evaluated using the Cochrane System Evaluator's Manual 5.1.0 RCT bias risk assessment tool. After evaluating, all studies achieved a score no less than 3, which indicating that all studies had good methodological quality. The summarized content for each study (13,20-29) is shown in Table 1.

\section{Meta-analysis results}

Total effective rate of safflower yellow pigment

The 12 studies $(13,20-29)$ that reported the effective rate were analyzed under a random effect model. Meta-analysis showed a significantly higher overall effective rate in safflower yellow group compared with that in the control group ( $\mathrm{RR}=1.24$, 95\% CI: 1.19, 1.30, $\mathrm{P}<0.00001$ ) (Figure 2) (30). There was no evidence of heterogeneity between these studies $\left(\mathrm{P}=0.009, \mathrm{I}^{2}=56 \%\right)$.

\section{Meta-regression analysis}

According to the results of the previous meta-analysis, heterogeneity exists among some studies (13,20-29) of safflower yellow in the treatment of cerebral infarction. In order to search for the causes of heterogeneity and the factors affecting the treatment effect, such as duration of treatment, age, gender, number of trials, and whether to use the blind method, a multivariate meta-regression analysis was performed for the age and the type of injection. The results are shown in Table 2.

It can be seen that the between-study heterogeneity tau ${ }^{2}$ $=0.001778$ is 0.006322 less than the previous 0.0081 after adding the two variables of age and type of injection. This indicates that different age and different types of injection production can explain $78.05 \%$ of the heterogeneity. Therefore, we considered the subgroup analysis of safflower yellow injection and safflower injection, which could effectively explain the heterogeneity and obtain reasonable conclusions.

\section{Total effective rate of safflower yellow injection}

Seven studies that reported the effective rate $(21,22,24-28)$ were analyzed under a fixed effect model. Meta-analysis showed a significantly higher effective rate in safflower yellow group compared with that in control group (OR $=4.33$, 95\% CI: 2.67, 7.05, $\mathrm{P}<0.00001$ ) (Figure 3). There was no evidence of heterogeneity between these studies $\left(\mathrm{P}=0.68, \mathrm{I}^{2}=0\right)$.

\section{Total effective rate of safflower injection}

Five studies that reported the effective rate $(13,20,23,29,30)$ were analyzed under a random effect model. Meta-analysis showed a significantly higher overall effective rate in safflower injection group compared with that in control group (OR $=6.00$, 95\% CI: 3.71, 9.72, $\mathrm{P}<0.00001)$ (Figure 4). There was no evidence of heterogeneity between these studies $(\mathrm{P}=0.03$, $\left.\mathrm{I}^{2}=62 \%\right)$.

\section{Detection bias}

The funnel plot of the total effective rate evaluated in the included papers $(13,20-29)$ was analyzed (Figure 5). The funnel plot was essentially symmetric, suggesting absence of publication bias (Egger $\mathrm{P}=0.223$; Begger $\mathrm{P}=0.2441$ ).

\section{Adverse reactions}

Six studies reported that no adverse reactions were observed (20-25); one did not mention adverse reactions (30); and five reported the occurrence of adverse reactions (26-30). The details of the inclusion of adverse reactions can be seen in Table 3. The above results suggested that safflower yellow injection and Sanqi Panax notoginseng injection had none or mostly mild adverse effects on patients.

\section{Pharmacoeconomic analysis}

\section{Cost calculation}

The cost variable for this paper was calculated from the perspective of patients. Drug prices understandably fluctuate throughout time, so the retail price of the therapy was chosen closest to the time of the study. Cost-effectiveness analysis does not distinguish anything based on the severity of disease. Direct medical cost, direct non-medical cost and indirect cost are the average cost of each disease state (mild, moderate and severe) (31). In this study, it was assumed that the direct nonmedical cost and indirect cost of the patients in each group were the same, respectively, and the difference in the total cost was caused by the direct medical cost. In addition, due to the similar costs of hospitalization and drug administration in direct medical costs, this study only considered the cost of the drug itself. When the treatment time of the disease exceeds one year, the cost should be discounted. According to the recommendation of the international pharmacoeconomic study, a $5 \%$ discount rate was adopted for cost data and uniformly discounts were applied to 2020.

In this study, the investigation of drug prices was 


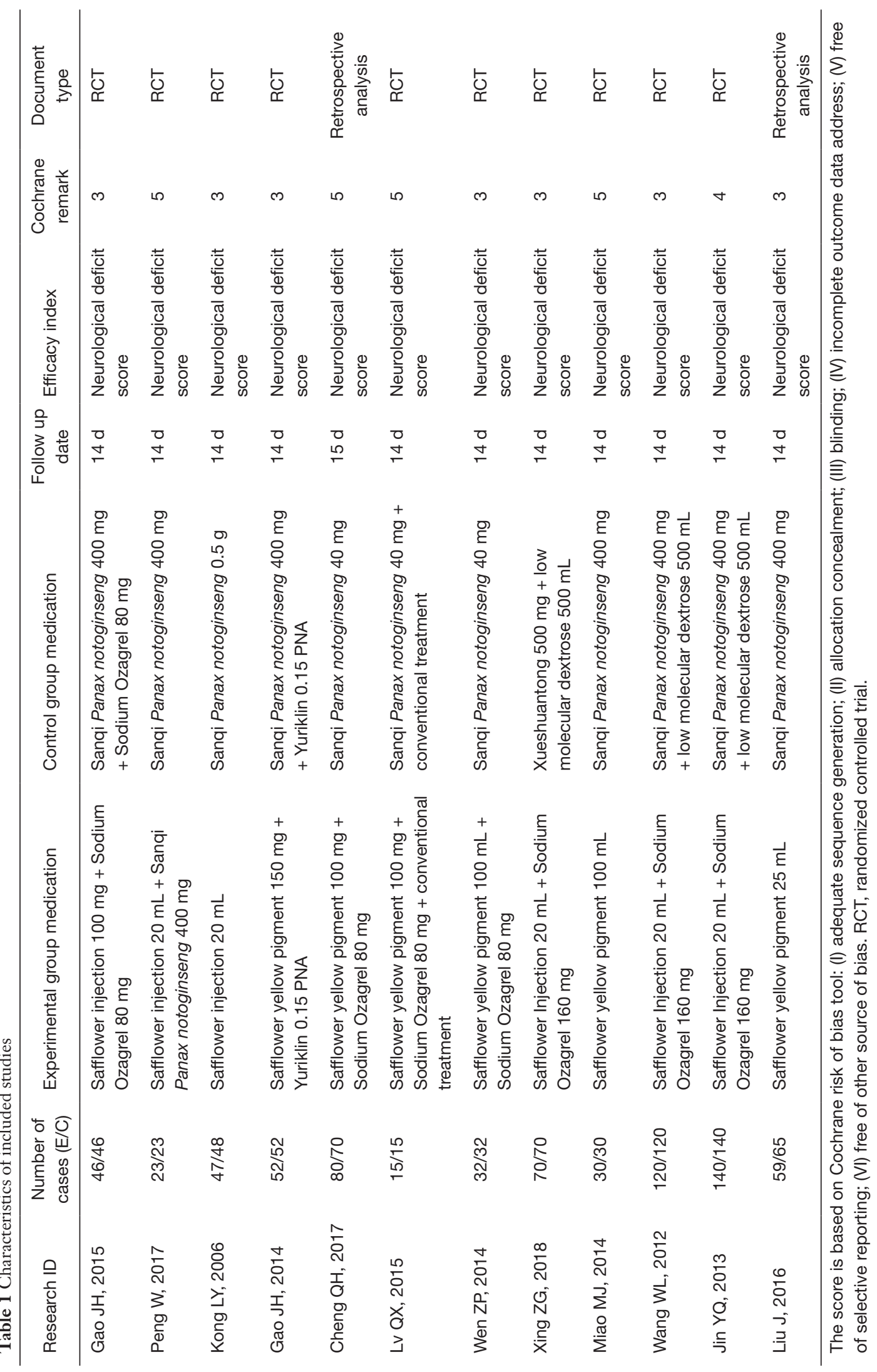




\begin{tabular}{|c|c|c|c|c|c|c|c|c|}
\hline Study or Subgroup & \multicolumn{2}{|c|}{ Experimental } & \multicolumn{2}{|c|}{ Control } & \multicolumn{2}{|r|}{ Risk Ratio } & $\begin{array}{c}\text { Risk Ratio } \\
\text { M-H, Fixed, } 95 \% \mathrm{Cl}\end{array}$ & \\
\hline Gao 2015 & 43 & 46 & 41 & 46 & $7.7 \%$ & $1.05[0.92,1.19]$ & - & \\
\hline Kong 2006 & 40 & $4 ?$ & 38 & 48 & $3.0 \%$ & $1.08[0.89,1.30]$ & & \\
\hline Liu 2016 & 51 & 59 & 52 & 65 & $9.2 \%$ & $1.08[0.92,1.27]$ & & \\
\hline Gao 2014 & 50 & 52 & 45 & 52 & $8.4 \%$ & $1.11[0.99,1.25]$ & & \\
\hline Lv 2015 & 13 & 15 & 11 & 15 & $2.1 \%$ & $1.18[0.82,1.70]$ & & \\
\hline $\operatorname{Jin} 2013$ & 133 & 140 & 106 & 140 & $19.8 \%$ & $1.25[1.13,1.39]$ & & \\
\hline Wang 2012 & 114 & 120 & 90 & 120 & $16.8 \%$ & $1.27[1.13,1.42]$ & & \\
\hline Xing 2018 & 69 & 30 & 53 & 30 & $9.9 \%$ & $1.30[1.14,1.49]$ & & \\
\hline Wen 2014 & 29 & 32 & 22 & 32 & $4.1 \%$ & $1.32[1.02,1.71]$ & & \\
\hline Chen 2017 & 75 & 80 & 48 & 70 & $9.6 \%$ & $1.37[1.16,1.62]$ & & \\
\hline Miao 2014 & 28 & 30 & 20 & 30 & $3.7 \%$ & $1.40[1.07,1.83]$ & & \\
\hline Peng 2017 & 22 & 23 & 9 & 23 & $1.7 \%$ & $2.44[1.46,4.10]$ & & \\
\hline Total $(95 \% \mathrm{Cl})$ & & 714 & & 711 & $100.0 \%$ & $1.24[1.19,1.30]$ & $\nabla$ & \\
\hline Total events & 667 & & 535 & & & & & \\
\hline $\begin{array}{l}\text { Heterogeneity: Chi } \\
\text { Test for overall effec }\end{array}$ & $\begin{array}{l}24.90, \mathrm{df} \\
Z=9.10\end{array}$ & $\begin{array}{l}11(P= \\
\approx 0.00\end{array}$ & $\begin{array}{l}0.009) ; 1 \\
001)\end{array}$ & $=569$ & & & $\begin{array}{ccr}0.7 & 1 & 1.5 \\
\text { erimental] } & \text { Favours }\end{array}$ & $\begin{array}{l}2 \\
\text { control] }\end{array}$ \\
\hline
\end{tabular}

Figure 2 Forest plot of overall effective rate in safflower yellow group compared with control group.

Table 2 Multivariate meta-regression analysis of year and type of injection

\begin{tabular}{lccccc}
\hline Logor & Coef. & Std.Err. & $\mathrm{t}$ & $\mathrm{P}>|\mathrm{t}|$ & $95 \% \mathrm{Cl}$ \\
\hline Year & 0.0190542 & 0.0124645 & 1.53 & 0.161 & $-0.0091425,0.0472508$ \\
Type & 0.1216314 & 0.0704028 & 1.73 & 0.118 & $-0.0376307,0.2808936$ \\
Cons & -38.24614 & 25.11704 & -1.52 & 0.162 & $-95.06482,18.57255$ \\
\hline
\end{tabular}

The variant type means safflower yellow injection of the different manufacturer. The proportion of between-study variance explained, $\mathrm{R}^{2}=0.6586$. Coef., regression coefficient; Std. Err., standard error.

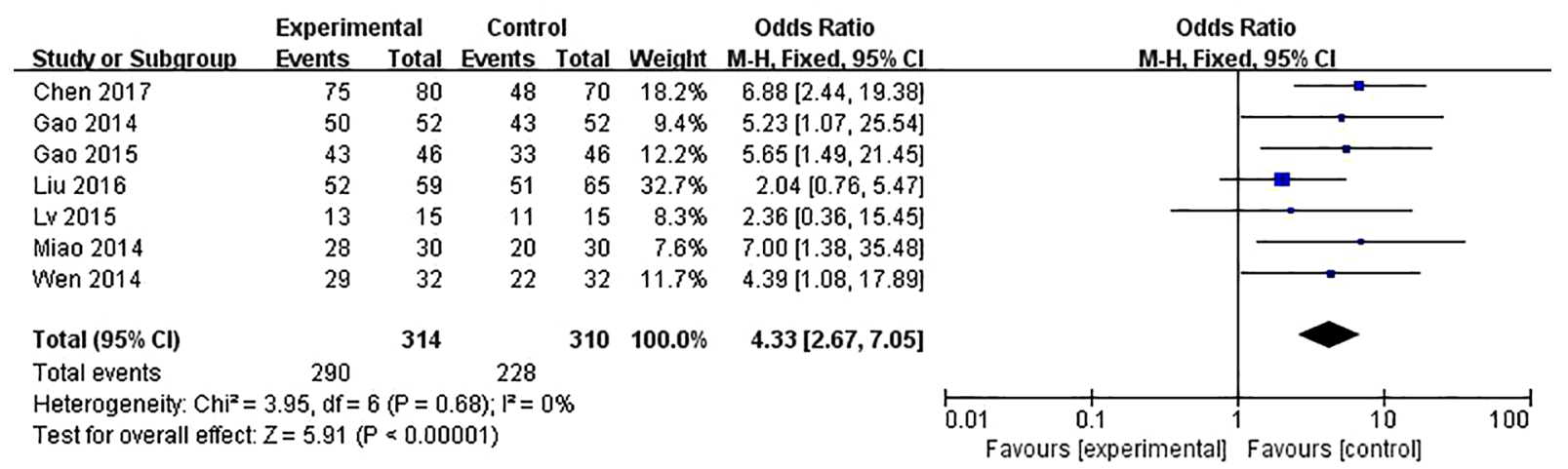

Figure 3 Forest plot of effective rate in safflower yellow group.

conducted through the Chinese 315 Drug Price Inquiry Network (https://www.315jiage.cn). The price of drugs used in the randomized controlled trials was included in the meta-analysis. According to the order of the price per milligram of active ingredient, the sensitivity analysis was conducted with the highest manufacturer retail price and the lowest manufacturer retail price. When calculating the drug cost of the both groups, the combined dose of the most used various drugs or injections in the literature was used as the drug cost of the treatment plan. The results showed that in the safflower yellow group, the treatment measures for calculating the cost were safflower yellow $100 \mathrm{mg}$ 


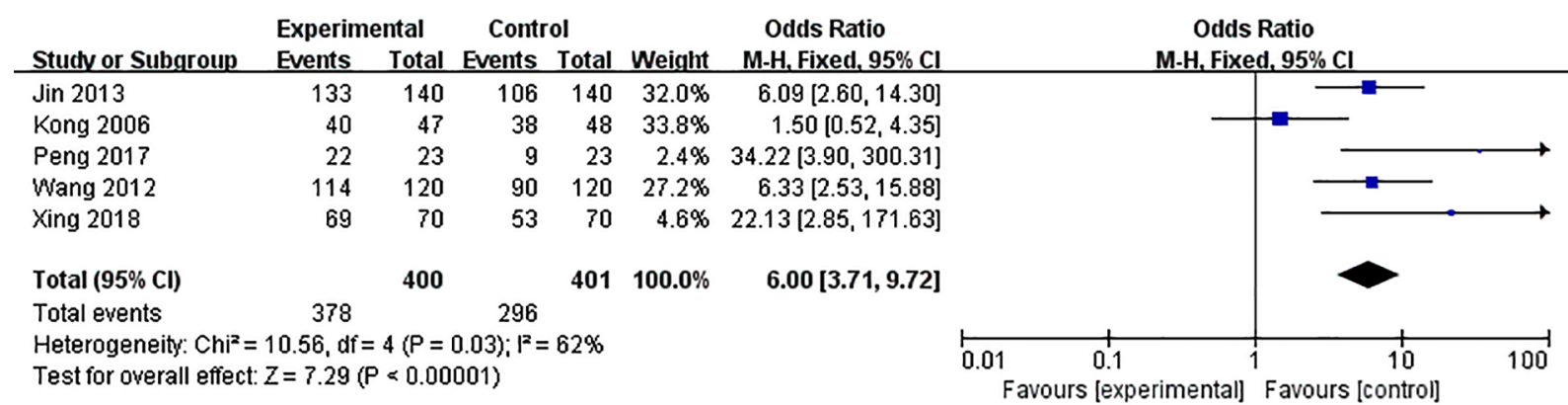

Figure 4 Forest plot of effective rate in safflower injection group.

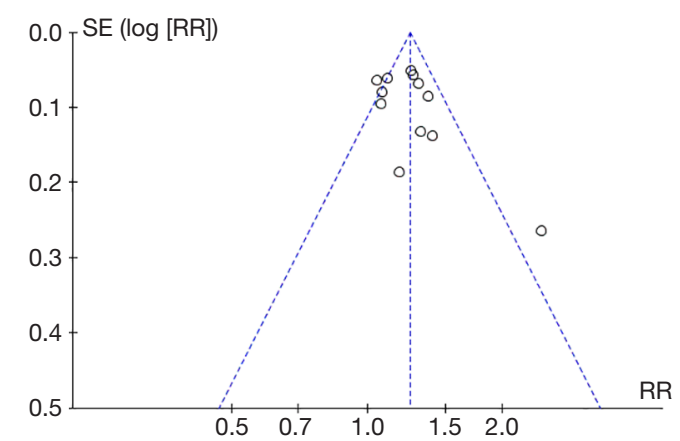

Figure 5 The funnel plot.

Table 3 Inclusion of adverse reactions

\begin{tabular}{|c|c|c|c|c|c|c|c|}
\hline $\begin{array}{l}\text { Adverse reactions } \\
\text { research ID }\end{array}$ & $\begin{array}{l}\text { Slight headache } \\
(E / C)\end{array}$ & $\begin{array}{l}\text { Sickness and } \\
\text { vomit }(E / C)\end{array}$ & $\begin{array}{l}\text { Loss of appetite } \\
\text { (E/C) }\end{array}$ & $\begin{array}{l}\text { Fever } \\
(\mathrm{E} / \mathrm{C})\end{array}$ & $\begin{array}{l}\text { Diarrhea } \\
(E / C)\end{array}$ & $\begin{array}{l}\text { Allergic reaction } \\
(E / C)\end{array}$ & $\begin{array}{c}\text { Incidence rate } \\
(\mathrm{E} / \mathrm{C})\end{array}$ \\
\hline Peng W, 2017 & $0 / 0$ & $1 / 0$ & $0 / 0$ & $0 / 0$ & $0 / 0$ & $1 / 3$ & $8.69 \% / 21.73 \%$ \\
\hline Lv XX, 2015 & $0 / 1$ & $0 / 0$ & $1 / 2$ & $0 / 1$ & $1 / 0$ & $0 / 0$ & $13 \% / 26 \%$ \\
\hline
\end{tabular}

E/C means experimental group/control group.

Table 4 Specific drug price chart

\begin{tabular}{llcc}
\hline Drug generic name & Cost & Maximum (yuan) & Minimum (yuan) \\
\hline Safflower yellow injection & 36.97 yuan $/ 50 \mathrm{mg} \times 1$ branch & 79.9 & 20.5 \\
Safflower injection & 8.95 yuan $/ 5 \mathrm{~mL} \times 1$ branch & 12.6 & 2.21 \\
Sanqi Panax notoginseng injection & 38.56 yuan $/ 400 \mathrm{mg} \times 1$ branch & 20 & 98 \\
Ozagrel sodium injection & 10 yuan/80 $\mathrm{mg} \times 1$ branch & 11.48 & 2 \\
\hline
\end{tabular}

Data sources: https://www.315jiage.cn. 
Table 5 Results of cost-effectiveness analysis of two treatment options

\begin{tabular}{lcccccc}
\hline Serial number & Treatment programs & Effect & Effect increment & Cost (yuan) & Cost increment (yuan) & ICER \\
\hline 1 & SPN group & $74.83 \%$ & - & 539.84 & - & - \\
2 & SYI group & $91.18 \%$ & 0.1635 & $1,175.16$ & 635.32 & $3,885.75$ \\
\hline
\end{tabular}

SPN, Sanqi Panax notoginseng; SYI, Safflower yellow injection; ICER, incremental cost-effectiveness ratio.

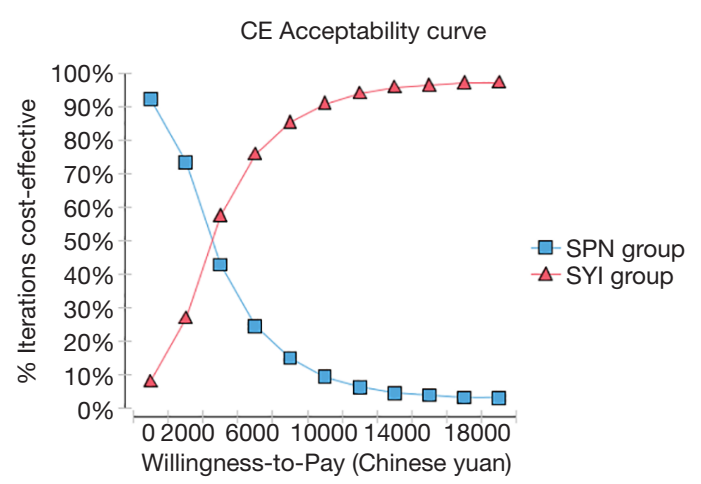

Figure 6 Cost-effective acceptance curve.

combined with ozagrel sodium $80 \mathrm{mg}$, and the total cost was 1,175.16 RMB (Chinese yuan). See Table 4 for specific drug prices.

\section{Cost effectiveness analysis}

In the case of limited treatment cycle, treatment dose and conventional treatment methods, a total of 12 articles were included in this study, which is the same number of articles included in the meta-analysis, with no article further excluded. According to the proportion of the studies shown in the forest chart in the Meta merger, the treatment efficacy of patients with angina pectoris in each study was weighted to obtain the effectiveness of treatment of cerebral infarction in each group. The results showed that the effective rate of the experiment group was $91.18 \%$, and the effective rate of the Sanqi Panax notoginseng group was $74.83 \%$, which was consistent with the outcome of the meta-analysis, indicating that the weighted calculations had good effectiveness, and could be included in the calculation of pharmacoeconomic evaluation. The results showed that the incremental cost-effectiveness ratio of the safflower yellow group was $3,885.75 \mathrm{RMB}$ with the Sanqi Panax notoginseng group as the control group. According to the recommendations of the World Health Organization on the evaluation of pharmacoeconomics, if ICER < GDP per capita, the increased cost is totally worthwhile; if GDP per capita $<$ ICER $<3$ times GDP per capita, the increased cost is acceptable; if ICER $>3$ times GDP per capita, the increased cost is not worth it (19)(30). In 2018, China's per capita GDP was 66,006 RMB (32), so the treatment plan in safflower yellow injection group is considered to be completely worthwhile and cost-effective. The detailed results are shown in Table 5.

\section{Sensitivity analysis}

(I) Two-factor sensitivity analysis: It was assumed that the treatment efficacy of the two groups fluctuated by $5 \%$; the cost was analyzed using the highest manufacturer retail price and the lowest manufacturer retail price. Assuming that the willingness to pay (WTP) is the GDP per capita in 2018, the whirlwind graph was obtained. The results showed that the parameter that had the greatest impact on the results was the treatment efficiency of the safflower yellow group. When the treatment efficiency of the safflower yellow group changed, the net benefit value of patients varied from 55,999.24 to 6,2018.99 RMB.

(II) Probability sensitivity analysis: Assumptions were declared that the effective rate was a beta distribution, and the cost was a triangular distribution. After each parameter was put into the TreeAge software, 1,000 second-order Monte Carlo simulations were performed, assuming that the patient's WTP changes ranged from 0 to 20,000 RMB. The cost-effective acceptance curve is shown in Figure 6. It can be seen that when WTP was less than 4,000 RMB, the Sanqi Panax notoginseng (SPN) group had a greater probability of forming a more economical program than the safflower yellow injection (SYI) group; when WTP was higher than 4,000 RMB, the probability of SYI forming a more economical program was greater than $50 \%$. That is, the SYI group is more cost-effective than the SPN group. The results of probability sensitivity analysis were consistent with the results of basic analysis, indicating that the results of basic analysis were robust. 


\section{Discussion}

Although Sanqi Panax notoginseng and safflower yellow have different mechanisms of action and main components, there are similarities in their pharmacological effects. It has been found through literature review that the relevant studies have been mostly conducted with small sample sizes, and some systematic reviews have been conducted previously to study the treatment of acute cerebral infarction with safflower yellow. No meta-analysis of safflower yellow injection and Sanqi Panax notoginseng injection exists in recent literature even though the therapeutic effects of safflower yellow injection and Sanqi Panax notoginseng injection on cerebral infarction can be analyzed and compared (8,33). Accordingly, several studies have been used to compare safflower yellow injection with Sanqi Panax notoginseng injection in treatment of cerebral infarction through meta-analysis.

In this work, the meta-analysis method was used to compare the effectiveness of injections of safflower yellow and Sanqi Panax notoginseng in the treatment of cerebral infarction, and the Bayesian method was used to compare the cost-effectiveness of the safflower yellow and Sanqi Panax notoginseng. A total of 12 studies were included in this systematic review (13,20-29). The baseline characteristics were compared and not statistically significant in each study. Meta-analysis showed that there was statistical difference in the total effectiveness between the experimental group and the control group when safflower yellow injection and Sanqi Panax notoginseng injection were used to treat acute cerebral infarction. This suggests that the adjuvant treatment using safflower yellow injection or Sanqi Panax notoginseng injection can improve the clinical effects of conventional treatment. From the perspective of treatment effects, using safflower yellow may be a better choice than the Sanqi Panax notoginseng. The evaluation indexes selected in this analysis were consistent, and the use of neurological deficit scores enhanced the rationality and comparability of the evaluation of treatment effects. In terms of safety, only six of the evaluated papers reported the occurrence of adverse reactions, and all of them were minor adverse reactions.

From the perspective of pharmacoeconomics, the ICER is $3,885.75 \mathrm{RMB}$, that is, the SYI group was more costeffective than SPN group, which is consistent with the result of cost-effectiveness analysis designed by Liu J in 2016. Thus, according to the result information of Sanqi Panax notoginseng injection and safflower yellow injection, we recommended that safflower yellow injection should be used to acute cerebral infarction patients priorly to Sanqi
Panax notoginseng injection in public hospitals.

The limitations of this study include: (I) the small sample size that was finally included in this study somewhat reduces the confidence or widespread applicability of this analysis; (II) the Cochrane score of the final literature reports included in this study is low, and there is lack of sufficient information to judge the research randomization, allocation concealment, blind methods, etc.; (III) the publications included in this study used different doses of safflower yellow pigment, and conventional treatments use different drugs, which may lead to selective bias; (IV) the literature included in this study are all published journal articles, absent of "gray literature", such as special reports, unpublished materials, etc.; (V) all the selected literature reported short-term studies without long-term follow-up monitoring, so the long-term safety and efficacy of safflower yellow treatment could not be evaluated.

\section{Conclusions}

In summary, the common use of safflower yellow injection and Sanqi Panax notoginseng injection in the treatment of acute cerebral infarction can improve the overall effectiveness of conventional treatment, and it is safe to use with few adverse reactions. At the same time, in case of high WTP, safflower yellow injection is more costeffective than Sanqi Panax notoginseng injection. Therefore, it is recommended that in the clinical treatment of acute cerebral infarction, safflower yellow pigment injection is used, and safflower yellow pigment injection can be combined with ozagrel sodium for injection within the economically acceptable range of most patients, which is inline with the cost of Sanqi Panax notoginseng. Considering the limited amount of data available for meta-analysis, it is suggested that more clinical trials be undertaken to verify these conclusions with more high-quality data, support further use of these formulations, and evaluate their longterm safety and efficacy profiles.

\section{Acknowledgments}

We appreciate Prof. C. Benjamin Naman of Ningbo University for careful language editing of the manuscript. Funding: None.

\section{Footnote}

Reporting Checklist: The authors have completed the 
PRISMA reporting checklist. Available at https://dx.doi. org/10.21037/atm-21-782

Peer Review File: Available at https://dx.doi.org/10.21037/ atm-21-782

Conflicts of Interest: All authors have completed the ICMJE uniform disclosure form (available at https://dx.doi. org/10.21037/atm-21-782). The authors have no conflicts of interest to declare.

Ethical Statement: The authors are accountable for all aspects of the work in ensuring that questions related to the accuracy or integrity of any part of the work are appropriately investigated and resolved. The study was conducted in accordance with the Declaration of Helsinki (as revised in 2013).

Open Access Statement: This is an Open Access article distributed in accordance with the Creative Commons Attribution-NonCommercial-NoDerivs 4.0 International License (CC BY-NC-ND 4.0), which permits the noncommercial replication and distribution of the article with the strict proviso that no changes or edits are made and the original work is properly cited (including links to both the formal publication through the relevant DOI and the license). See: https://creativecommons.org/licenses/by-nc-nd/4.0/.

\section{References}

1. Li LJ, Li YM, Qiao BY, et al. The Value of Safflower Yellow Injection for the Treatment of Acute Cerebral Infarction: A Randomized Controlled Trial. Evid Based Complement Alternat Med 2015;2015:478793.

2. Li YY, Liu SS, Sun XD, et al. Meta-analysis of safflower yellow injection in the treatment of acute cerebral infarction. Chinese Traditional Patent Medicine 2019;6:1472-5.

3. Li LJ. Clinical study of safflower yellow injection in the treatment of acute cerebral infarction. Journal of Beijing University of Chinese Medicine 2008;15:1-4.

4. Zhang H, Wang YL, Bai B, et al. Research on the impact of medical reform policies on hospitalization costs of patients with cerebral infarction. Chinese Journal of Stroke 2016;11:607-12.

5. Department of Disease Control, Ministry of Health, Neurology Branch of Chinese Medical Association: Guidelines for the prevention and treatment of cerebrovascular diseases in China. Beijing: People's Medical Publishing House, 2007.

6. Lu AK, Zheng HY, Wu S, et al. Influencing mechanism and intervention strategy of mental resilience in patients with cerebral infarction. Chongqing Medicine 2017;46:1370-2.

7. Zhou XB, Xu K. Advances in research on protective mechanism of Panax notoginseng saponins on cerebral ischemia/reperfusion injury. Medical Repeat Journal 2008;24:3784-6.

8. Li ZW, Li XM. Clinical application of safflor yellow in cardiovascular system. Shenyang Force Medicine 2012;2:155-7.

9. Du S, Deng Y, Yuan H, et al. Safflower Yellow B Protects Brain against Cerebral Ischemia Reperfusion Injury through AMPK/NF-kB Pathway. Evid Based Complement Alternat Med 2019;2019:7219740.

10. Zheng WC. Effect and mechanism of safflor yellow on myocardial ischemia-reperfusion model in rats. Chinese Pharmacological Bulletin 2003;19:1032-4.

11. Chai ZL,Chen YN. Effect of safflower injection on blood viscosity, platelet and anticoagulant function in patients with pulmonary heart disease. Journal of Pharmaceutical Practice 2003;21:260-3.

12. Liao H,Shi YH. Effect of Safflower Injection on Hemorheology of Cardiovascular and Cerebrovascular Diseases. China Journal of Traditional Chinese Medicine and Information 2003;9:15-6.

13. Wang YF, Zhai WJ. Clinical Observation of Honghua Injection in Treating Coronary Heart Disease with Angina Pectoris and Myocardial Ischemia. Medical Theory and Practice 2003;9:1002-3.

14. Jie LQ, Han GX. Advances in Research on Traditional Chinese Medicine and Western Medicine for Acute Ischemic Stroke. Chinese Journal of Convalescent Medicine 2019;10:1047-50.

15. Moher D, Liberati A, Tetzlaff J, et al. Preferred reporting items for systematic reviews and meta-analyses: the PRISMA statement. PLoS Med 2009;6:e1000097.

16. Goodyear MD, Krleza-Jeric K, Lemmens T. The Declaration of Helsinki. BMJ 2007;335:624-5.

17. Higgins J. Cochrane Handbook for Systematic Reviews of Interventions Version 5.1.0. 2011. Available online: https:// handbook-5-1.cochrane.org/

18. Guan X, Li HC, Shao RJ, et al. A systematic review and economic evaluation of one-week esomeprazole-based triple therapy versus omeprazole-based triple therapy for patients with peptic ulcer. Chinese Journal of Hospital 
Pharmacy 2019;39:502-7.

19. Sun LH. Pharmacoeconomics. Beijing: China Medical Science and Technology Press 2004;2:1-177.

20. Wang WL. Efficacy of ozagrel sodium combined with Honghua injection in the treatment of cerebral infarction. Chinese Community Physician Medical Major 2012;14:27-8.

21. Gao JH. Clinical observation of ozagrel sodium combined with traditional Chinese medicine preparation in treating cerebral infarction. Guide of Chinese Medicine 2015;17:63-4.

22. Miao MJ, Chen ZB. Clinical observation of 30 cases of acute cerebral infarction treated with safflower yellow pigment injection. Medicine Information 2014;3:109-10.

23. Xing ZG. Therapeutic effect of combined treatment with ozagrel sodium and safflower injection on cerebral infarction. Health Literature 2018;6:230.

24. Gao JH, Yu LL. Clinical observation of equuklin combined with traditional Chinese medicine preparation in treating cerebral infarction. Guide of China Medicine 2014;30:192-3.

25. Liu J. Cost-effectiveness analysis of traditional Chinese medicine injection in treating acute cerebral infarction. Tianjin Pharmacy 2016;8:813-5.

26. Chen QH. Adverse reactions and therapeutic effects

Cite this article as: Li Y, Lin Y, Shi Z, Hu Y, Shao T, Li M, Zhu J, Sun L, Chang F. Safflower yellow pigment and Sanqi Panax notoginseng in the treatment of acute cerebral infarction: a systematic review, meta-analysis, and cost-effectiveness analysis. Ann Transl Med 2021;9(18):1407. doi: 10.21037/atm-21-782 of sodium ozagrel combined with safflower yellow in the treatment of cerebral infarction. Mod Diagn Treat 2017;23:4363-4.

27. Lv XX. Clinical efficacy of sodium ozagrel combined with safflower yellow in the treatment of cerebral infarction. China's Rural Health 2015;18:6-7.

28. Wen ZP. Clinical observation of ozagrel sodium combined with safflower yellow in the treatment of cerebral infarction. Mod Diagn Treat 2014;22:5127-8.

29. Peng W, Wang YM, Du XY. Treatment of 46 cases of acute ischemic stroke with Honghua injection. Gansu Science and Technology 2017;4:88-9.

30. Jin YQ. Therapeutic effect of combined treatment with ozagrel sodium and safflower injection on cerebral infarction. Seek Medical and Ask For Medicine 2013;3:153-4

31. Peng XX, Yan LX, Liu C, et al. Benign disease prone to be misdiagnosed as malignant pulmonary nodules: Minute meningothelioid nodules. Thorac Cancer 2019;10:1182-7.

32. National Bureau of Statistics. Accessed 5th May 2020. Available online: http://www.stats.gov.cn/tjsj/

33. Du HY, Du XH. Rat nerve growth factor combined with safflorin in the treatment of senile diabetes complicated with cerebral infarction. Journal of Kunming University 2016;37:84-7. 